\title{
High-energy collective electronic excitations in layered transition-metal dichalcogenides
}

\author{
Pierluigi Cudazzo, ${ }^{1,2}$ Kari O. Ruotsalainen,${ }^{3}$ Christoph J. Sahle, ${ }^{3}$ Ali Al-Zein, ${ }^{4}$ Helmuth Berger, ${ }^{5}$ Efrén Navarro-Moratalla, ${ }^{6}$ \\ Simo Huotari, ${ }^{3}$ Matteo Gatti, ${ }^{2,7,8}$ and Angel Rubio ${ }^{1,2}$ \\ ${ }^{1}$ Nano-Bio Spectroscopy Group, Departamento Física de Materiales, Universidad del País Vasco, Centro de Física de Materiales \\ CSIC-UPV/EHU-MPC and DIPC, Avenida Tolosa 72, E-20018 San Sebastián, Spain \\ ${ }^{2}$ European Theoretical Spectroscopy Facility (ETSF) \\ ${ }^{3}$ Department of Physics, P.O. Box 64, FI-00014 University of Helsinki, Helsinki, Finland \\ ${ }^{4}$ European Synchrotron Radiation Facility, B.P. 220, F-38043 Grenoble Cedex, France \\ ${ }^{5}$ École Polytechnique Fédérale de Lausanne (EPFL), Institut de Physique des Nanostructures, CH-1015 Lausanne, Switzerland \\ ${ }^{6}$ Instituto de Ciencia Molecular (ICMol), Universidad Valencia, Valencia, Spain \\ ${ }^{7}$ Laboratoire des Solides Irradiés, École Polytechnique, CNRS-CEA/DSM, F-91128 Palaiseau, France \\ ${ }^{8}$ Synchrotron SOLEIL, L'Orme des Merisiers, Saint-Aubin, B.P. 48, F-91192 Gif-sur-Yvette, France \\ (Received 29 May 2014; revised manuscript received 25 August 2014; published 16 September 2014)
}

\begin{abstract}
We characterize experimentally and theoretically the collective electronic excitations in two prototypical layered transition-metal dichalcogenides, $\mathrm{NbSe}_{2}$ and $\mathrm{Cu}_{0.2} \mathrm{NbS}_{2}$. The energy- and momentum-dependent dynamical structure factor was measured by inelastic x-ray scattering (IXS) spectroscopy and simulated by time-dependent density-functional theory. We find good agreement between theory and experiment, provided that $\mathrm{Nb}$ semicore states are taken into account together with crystal local-field effects. Both materials have very similar spectra, characterized by two main plasmons at 9 and $23 \mathrm{eV}$, which we show to both have $\pi+\sigma$ character on the basis of a detailed analysis of the band structure. Finally, we discuss the role of the layer anisotropy in the dispersion of these plasmons.
\end{abstract}

DOI: 10.1103/PhysRevB.90.125125

PACS number(s): 71.45.Gm, 78.70.Ck, 73.21.Ac

\section{INTRODUCTION}

Layered materials like graphite consist of quasi-twodimensional sheets bound together by weak van der Waals forces. This peculiarity together with the recent advances in nanoscale growth and mechanical exfoliation allow isolating single sheets to fabricate low-dimensional systems such as zero-dimensional quantum dots or nanoparticles, onedimensional nanoribbons or nanotubes, and two-dimensional nanosheets [1-3]. This makes these materials interesting not only for practical applications but also for fundamental understanding of collective excitations in confined geometries. When the dimensionality is reduced, the inherent increase in the relative importance of Coulomb interactions together with strongly reduced screening imply that many-body effects become particularly prominent, giving rise to new phenomena that cannot be observed in standard three-dimensional (3D) bulk systems. The interest in layered materials grew rapidly after the synthesis of graphene [4]. In fact, even if its unique electronic properties make this system one of the most promising materials for future carbon-based electronics, the lack of band gap in the electronic band structure $[5,6]$ makes the creation of graphene nanodevices highly nontrivial. This stimulated the search for other families of semiconducting layered materials. Among them, transition metal dichalcogenides (TMD) [7] are probably the materials with the most versatile electronic properties [8,9]. Their chemical formula is $\mathrm{MX}_{2}$, where $\mathrm{M}$ is a transition metal and $\mathrm{X}$ a chalcogen $(\mathrm{S}, \mathrm{Se}$, and $\mathrm{Te})$. In general, their crystal structures are such that each hexagonally packed sheet is characterized by a layer of transition metal atoms embedded between two layers of chalcogen atoms. Atoms within a sheet are bonded covalently, while individual sheets are bound via weak van der Waals interaction, which makes the properties of these materials very anisotropic as in graphite. Depending on the transition metal $\mathrm{M}$, they display metallic or insulating behavior [7]. Metallic systems as for example $\mathrm{NbSe}_{2}$ and $\mathrm{TaS}(\mathrm{Se})_{2}$ exhibit remarkable low-temperature phenomena, including the competition between superconductivity and charge-density wave order [10]. Insulators like $\mathrm{MoS}_{2}$, on the other hand, are indirect band gap materials in the bulk form. However, in two dimensions they display a direct band gap [11,12], becoming promising materials for optoelectronic applications [13]. An essential contribution to gain insight into the electronic structure of these materials, for both applications and fundamental problems, can be obtained by studying the elementary excitations that characterize their electronic properties. In the present work, we therefore investigate the dynamical response of TMD belonging to the $2 \mathrm{H}$ family with the aim of understanding the nature of the neutral collective excitations in these systems. Neutral excitations (plasmons in particular) have been extensively investigated in the past using electron energy loss spectroscopy (EELS) [14-16]. These experiments showed that the loss function of TMD is similar to that of graphite. In fact, for both metals and insulators, it is possible to identify two plasmons in the EELS spectra measured for in-plane momentum transfer: a low-energy plasmon at about $9 \mathrm{eV}$ and a high-energy plasmon involving all valence electrons at about $23 \mathrm{eV}$. Beside these two features, metallic TMD present also an intraband plasmon at about $1 \mathrm{eV}$ involving only the charge-carrier electrons [17-20]. However, neutral excitations in these materials have never been studied at large momentum transfer where the short-range part of the Coulomb interaction plays a key role. Only recent first-principle calculations investigated the behavior of the intraband plasmon at large momentum transfer normal to the layers, showing that this collective excitation has a periodic 
behavior with the reappearance around Bragg reflections of the plasmon of the first Brillouin zone [20]. This can be interpreted as a clear effect of short-range charge fluctuations induced by crystal local fields [21]. Nevertheless, the dispersion, i.e., the energy-momentum relation, of the high energy plasmons is a topic that to the best of our knowledge has not been investigated so far. Inelastic x-ray scattering (IXS) spectroscopy allows measuring the dynamical structure factor, which is related to the imaginary part of the inverse dielectric function. Contrarily to EELS, IXS is particularly suitable for experiments at large momentum transfer out of the first Brillouin zone. In this way it is possible to probe the plasmon dispersion and look at the effects of the short-range part of the Coulomb interaction, which becomes more and more important as the momentum transfer increases. In the following, combining IXS experiments and first-principle calculations based on time-dependent densityfunctional theory (TDDFT) [22], we present an analysis of the high-energy collective excitations and their dispersion for two prototypical metallic TMD, namely $2 \mathrm{H}-\mathrm{Cu}_{0.2} \mathrm{NbS}_{2}$ and $2 \mathrm{H}-\mathrm{NbSe}_{2}$. In $\mathrm{NbSe}_{2}$ the critical temperature for the phase transition to the superconducting state is $7.2 \mathrm{~K}$, and for the charge density wave ordered phase it is $33.5 \mathrm{~K}[23] . \mathrm{Cu}_{0.2} \mathrm{NbS}_{2}$ is superconducting below $2.5 \mathrm{~K}$ [24,25] but to our knowledge whether charge density wave order exists has not been studied.

\section{METHODS}

\section{A. Experiment}

The experiment was performed at the beamline ID20 of the European Synchrotron Radiation Facility. The radiation was produced utilizing three undulators with $32-\mathrm{mm}$ period. The incident energy was selected with a $\mathrm{Si}(111)$ monochromator. The beam was focused to a spot of $15 \mu \mathrm{m} \times 15 \mu \mathrm{m}(H \times V)$ on the samples using Kirkpatrick-Baez mirrors. A Johann-type diced $\mathrm{Si}(533)$ analyzer with a $0.7 \times 0.7 \mathrm{~mm}^{2}$ cube size was utilized. The analyzer was masked leaving an active area of $20 \times 60(H \times V) \mathrm{mm}^{2}$ to reach a momentum resolution of $\approx 0.1 \AA^{-1}$. The analyzer bending radius was $1 \mathrm{~m}$. The analyzer focused the scattered radiation onto a Maxipix detector. The monochromator and spectrometer bandpasses summed up to an energy resolution of $1 \mathrm{eV}$.

The samples were thin platelike crystals of $\mathrm{NbSe}_{2}$ and $\mathrm{Cu}_{0.2} \mathrm{NbS}_{2} . \mathrm{NbSe}_{2}$ and $\mathrm{Cu}_{0.2} \mathrm{NbS}_{2}$ powders were prepared starting from constituting elements at stoichiometric ratio, and the crystals were grown by thermal gradient using iodine as a transport agent. Details of the crystal growth are described in Ref. [26]. Sample stoichiometry was verified by standardless energy dispersive spectrometry using a Jeol JXA-8600 electron probe microanalyzer. Approximate dimensions of the samples were $10 \times 5 \times 0.1 \mathrm{~mm}^{3}$. The samples were mounted on a goniometer so that the $c$ axis was in the scattering plane and the sample orientation was determined using x-ray diffraction. The $c$-axis lattice parameters were 12.54 and $12.11 \AA$ for $\mathrm{NbSe}_{2}$ and $\mathrm{Cu}_{0.2} \mathrm{NbS}_{2}$, respectively. The $\mathrm{Cu}_{0.2} \mathrm{NbS}_{2}$ sample was then further tilted from the scattering plane by $\approx 3^{\circ}$ to reduce the background from Bragg peaks and phonon scattering when $\mathbf{q}$ was close to a reciprocal lattice point or along a symmetry line. Similarly the $\mathrm{NbSe}_{2}$ was also tilted by $\approx 1.5^{\circ}$. The energy loss spectra were recorded with the
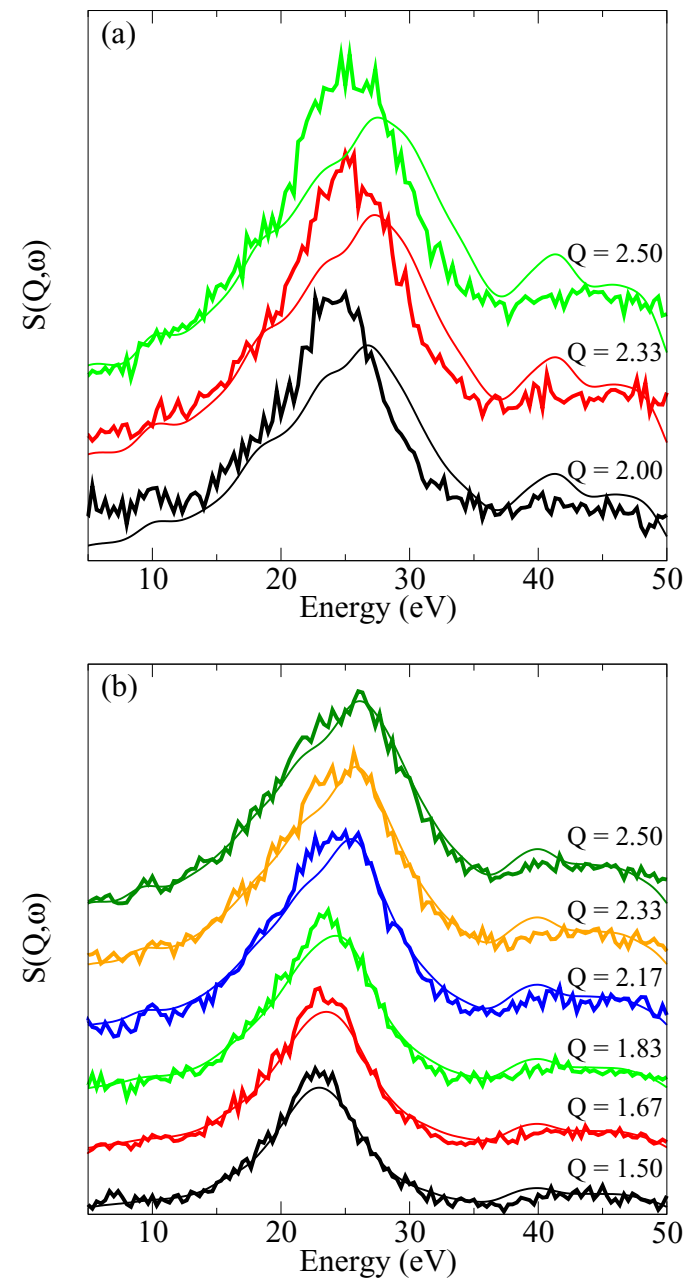

FIG. 1. (Color online) Comparison between experimental (bold line) and theoretical (thin line) spectra of $\mathrm{Cu}_{0.2} \mathrm{NbS}_{2}$ (a) and $\mathrm{NbSe}_{2}$ (b) evaluated at $\mathbf{Q}$ (in multiples of $2 \pi / c$ ) along the $c$ axis with a broadening of $1.0 \mathrm{eV}$.

inverse energy scan technique. The analyzer Bragg angle was kept fixed at $86^{\circ}$ and the incident energy was scanned from -2 to $+50 \mathrm{eV}$ about a nominal incident energy of $7504.2 \mathrm{eV}$. See Fig. 1 for the momentum transfer values. The spectra were normalized with an incident intensity monitor signal. For each value of $\mathbf{q}$, the spectra were measured repeatedly. The measured spectra were checked for internal consistency after the above-mentioned normalization, and finally averaged to yield the final spectrum. An empirically determined constant background was subtracted from the results, which were finally normalized to have the same area as the theoretical $S(\mathbf{q}, \omega)$ in the $\omega$ interval $10-40 \mathrm{eV}$.

\section{B. Computational details}

The microscopic dielectric function $\epsilon$ is related to the susceptibility $\chi$ (Ref. [27]) by the relation $\epsilon^{-1}=1+v \chi$ ( $v$ being the Coulomb potential). In TDDFT $\chi$ is the solution of the Dyson-like equation: $\chi=\chi_{0}+\chi_{0}\left(v+f_{x c}\right) \chi$, where $\chi_{0}$ is the Kohn-Sham (KS) susceptibility expressed in terms of KS eigenenergies and eigenfunctions, while $f_{x c}$ is the exchangecorrelation kernel, for which we use the adiabatic local-density 
approximation (ALDA). When $f_{x c}=0$ we retrieve the random phase approximation (RPA). The Fourier components of both $\chi$ and $\epsilon$ are matrices in terms of the reciprocal lattice vectors $\mathbf{G}$. The macroscopic dielectric function $\epsilon_{M}$ is given by $\epsilon_{M}(\mathbf{Q}, \omega)=1 / \epsilon_{\mathbf{G G}}^{-1}(\mathbf{q}, \omega)$, where $\mathbf{Q}=\mathbf{q}+\mathbf{G}$ and $\mathbf{q}$ is inside the first Brillouin zone. The dynamical structure factor $S(\mathbf{Q}, \omega)$ measured in IXS experiments is directly related to the loss function, i.e., the imaginary part of the inverse macroscopic dielectric function, through the following equation:

$$
S(\mathbf{Q}, \omega)=-\frac{\hbar|\mathbf{Q}|^{2}}{4 \pi^{2} e^{2} n_{0}} \operatorname{Im} \epsilon_{M}^{-1}(\mathbf{Q}, \omega),
$$

where $n_{0}$ denotes the average electron density. In the present work the KS eigenenergies and eigenfunctions used to determine $\chi_{0}$ have been evaluated in the local-density approximation (LDA) implemented in a plane-wave-based code [28]. In our calculations we adopt the experimental lattice structures [29]. We use Troullier-Martins and Hartwigsen-GoedeckerHutter norm-conserving pseudopotentials [30] (with an energy cutoff of $120 \mathrm{Ry}$ ) where $\mathrm{Nb} 4 s 4 p$ semicore states are not pseudized but explicitly taken into account. In the calculation of $\chi_{0}$ (Ref. [31]) we used a $24 \times 24 \times 12$ grid of $\mathbf{k}$ points and included 200 bands. The macroscopic dielectric function has been obtained by inverting a matrix of $300 \mathbf{G}$ vectors (those parameters lead to converged results for the response function in the range of energies and momentum studied in the present work). Finally, the electron doping induced in the $\mathrm{NbS}_{2}$ by the $\mathrm{Cu}$ atoms has been simulated by shifting upward the Fermi level according to a simple rigid-band model.

\section{RESULTS AND DISCUSSIONS}

In Figs. 1(a) and 1(b) we compare the experimental spectra of $\mathrm{Cu}_{0.2} \mathrm{NbS}_{2}$ and $\mathrm{NbSe}_{2}$ obtained from IXS measurements with the theoretical ones evaluated in ALDA for momentum transfer normal to the layers. Through TDDFT calculations, these TMD were found to be characterised by very similar dynamical response functions at low energies [20]. This is now confirmed also in a higher-energy range by the present results. In both systems the experimental loss function is dominated by a wide peak centered at about $23 \mathrm{eV}$ which is well reproduced by the calculations. Simulated spectra display a blueshift of this main peak of at most $\sim 2 \mathrm{eV}$ with respect to experiments. Besides possible effects of $\mathrm{Cu}$ doping beyond a rigid-band model and the slight discrepancy between the lattice parameters of Ref. [29] and those of the actual samples [32], the agreement between theory and experiment could be improved by taking into account the effect of the finite lifetimes of the electrons and holes involved in the excitations. They are neglected in our approach and have been shown to cause a redshift of the plasmon energy, especially at large momentum transfer [33-35]. In the energy window between 5 and $50 \mathrm{eV}$ we can identify two other features at about 9 and $40 \mathrm{eV}$, which are visible in both theoretical and experimental spectra. However, only the large peak at $23 \mathrm{eV}$ corresponds to a zero of $\operatorname{Re} \epsilon_{M}(\mathbf{Q}, \omega)$ and it can be thus interpreted as a collective excitation of all valence electrons. The other two features are instead associated to interband transitions. Finally, also the behavior of the spectra as a function of the

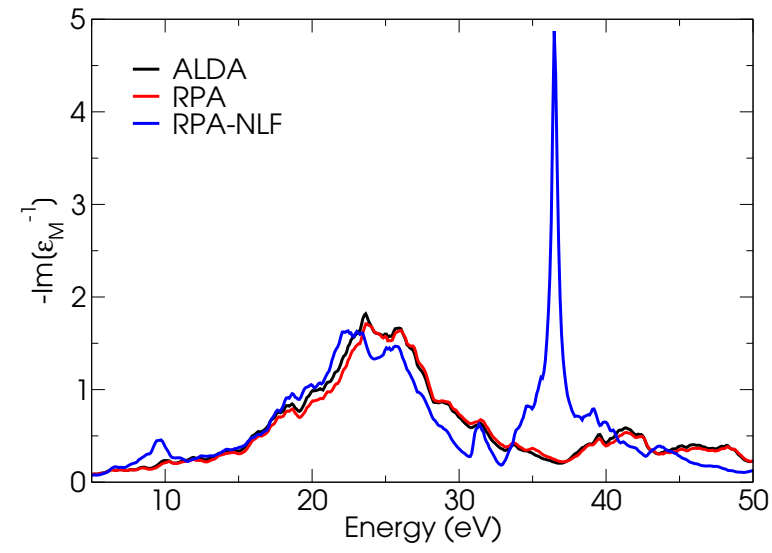

FIG. 2. (Color online) Comparison between the theoretical spectra (loss function) of $\mathrm{Cu}_{0.2} \mathrm{NbS}_{2}$ at $\mathbf{Q}=0.77 \AA^{-1}$ along the $c$ axis evaluated in different approximations. In this calculations we have used a broadening of $0.1 \mathrm{eV}$.

momentum transfer is well reproduced by our calculation. In fact, in agreement with experiments we find that in the range $0.77 \leqslant|\mathbf{Q}| \leqslant 1.29 \AA^{-1}$, the large peak at $23 \mathrm{eV}$ broadens but does not disperse appreciably in energy. The overall agreement is sufficiently good to allow us to use the results of the calculation to perform an analysis of the spectra. In general, the plasmon behavior is the result of several competing effects related to the electronic band structure, crystal local fields, and the exchange correlation, which in our approach is described by the $f_{x c}$ kernel [27]. To understand the role played by each contribution, we compare in Fig. 2 the loss functions of $\mathrm{Cu}_{0.2} \mathrm{NbS}_{2}$ evaluated in different approximations: ALDA, RPA, and RPA without local fields (called RPA-NLF in Fig. 2). When both local fields and exchange-correlation effects are neglected (blue line), we find that, in addition to the main plasmon peak at $23 \mathrm{eV}$, the loss function is characterized by a sharp peak at $36 \mathrm{eV}$ which arises from independent electron-hole pairs involving $\mathrm{Nb} 4 p$ semicore states. The inclusion of the crystal local fields causes a global reduction of the oscillator strength of the spectrum (compare blue and red lines in Fig. 2). In particular the semicore sharp peak at $36 \mathrm{eV}$ is largely suppressed and blueshifted to $40 \mathrm{eV}$. This strong depolarization effect induced by the crystal local fields on the semicore peak is due to the localized nature of these states.

In fact, crystal local-field effects (LFE) are related to the inhomogeneity of the induced electronic charge (thus appearing in the induced Hartree potential). Semicore states are spatially strongly localized and quite polarizable, which explains the relevance of LFE [36] and shows that LFE cannot be neglected [37]. Finally, from the comparison of the spectra evaluated in ALDA and RPA we can conclude that the effect of the ALDA $f_{x c}$ kernel is small since it causes only a redshift of the plasmon peak of about $0.3 \mathrm{eV}$. This in the following allows us to neglect the kernel and interpret the spectra in RPA. Highenergy interband transitions due to $\mathrm{Nb}$ semicore states play a crucial role also for the main plasmon at lower frequency [38]. In fact, when they are removed in the RPA-NLF calculation the main plasmon at $23 \mathrm{eV}$ is blueshifted by $1 \mathrm{eV}$ (compare blue 


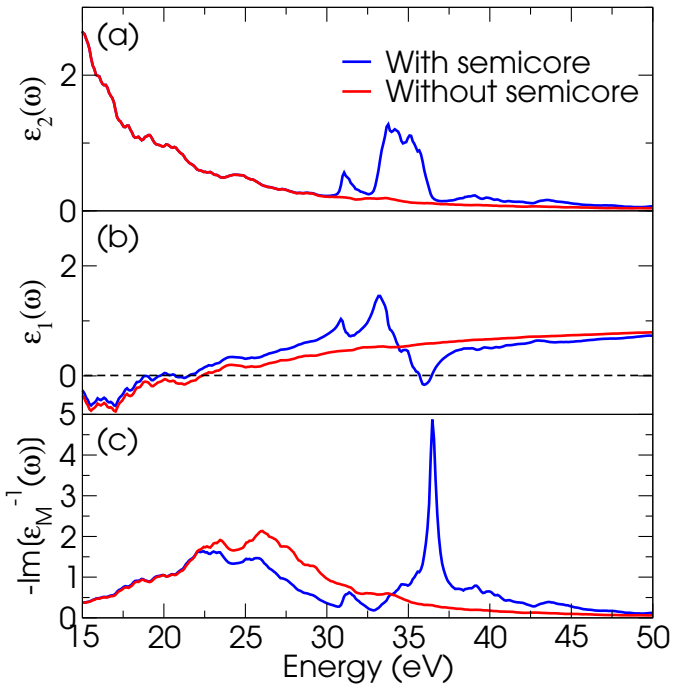

FIG. 3. (Color online) (a) Imaginary and (b) real part of the dielectric function and (c) loss function of $\mathrm{Cu}_{0.2} \mathrm{NbS}_{2}$ at $\mathbf{Q}=0.77 \AA^{-1}$ along the $c$ axis evaluated in RPA-NLF with and without semicore states with a broadening of $0.1 \mathrm{eV}$.

and red lines in the bottom panel of Fig. 3). The excitation of semicore states is seen as an additional double peak in $\epsilon_{2}=\operatorname{Im} \epsilon_{M}$ at $\sim 32-34 \mathrm{eV}$ (see top-most panel in Fig. 3). This additional peak in $\epsilon_{2}$ through the Kramers-Kronig relation affects $\epsilon_{1}=\operatorname{Re} \epsilon_{M}$ in a wide energy range (see middle panel in Fig. 3) changing the position of its zero crossing and hence of the main plasmon. We thus conclude that $\mathrm{Nb}$ semicore electrons effectively screen the main plasmon. When LFE are taken into account, this screening effect of $\mathrm{Nb}$ semicore states is strongly reduced and the main plasmon is blueshifted (see Fig. 2). Thus, crystal local fields have two effects: a direct one, which consists of suppressing the oscillator strength, and an indirect one through the semicore states which modifies the position the plasmon peak. To better illustrate the plasmon dispersion, we show in Fig. 4 the RPA loss function evaluated in a larger window of momentum transfer between 0.39 and $1.00 \AA^{-1}$. The plasmon is characterized by an oscillating trend as function of $\mathbf{Q}$ within a small energy range $\sim 23-24 \mathrm{eV}$. Between 0.39 and $0.60 \AA^{-1}$ the plasmon dispersion is positive. Then it becomes negative up to $0.77 \AA^{-1}$. Finally at larger $\mathbf{Q}$ it goes back to being positive. The negative dispersion is related to the appearance of a new feature at $26 \mathrm{eV}$ that increases in intensity as the momentum transfer increases [39]. This peak, related to interband transitions above the plasmon frequency that are dipole forbidden at small momentum transfer but are activated by multipole terms, screens the plasmon causing a redshift of the peak that is stronger as $\mathbf{Q}$ is larger. Finally at $\mathbf{Q}=0.77 \AA^{-1}$ the new feature starts to disperse positively and its effect on the plasmon becomes weaker: the slope of the plasmon dispersion switches to positive. Thus, the behavior of the valence plasmon is the result of two competing effects: the intrinsic positive dispersion related to the collective nature of the excitation and the appearance of higher energy interband transitions at large momentum transfer.

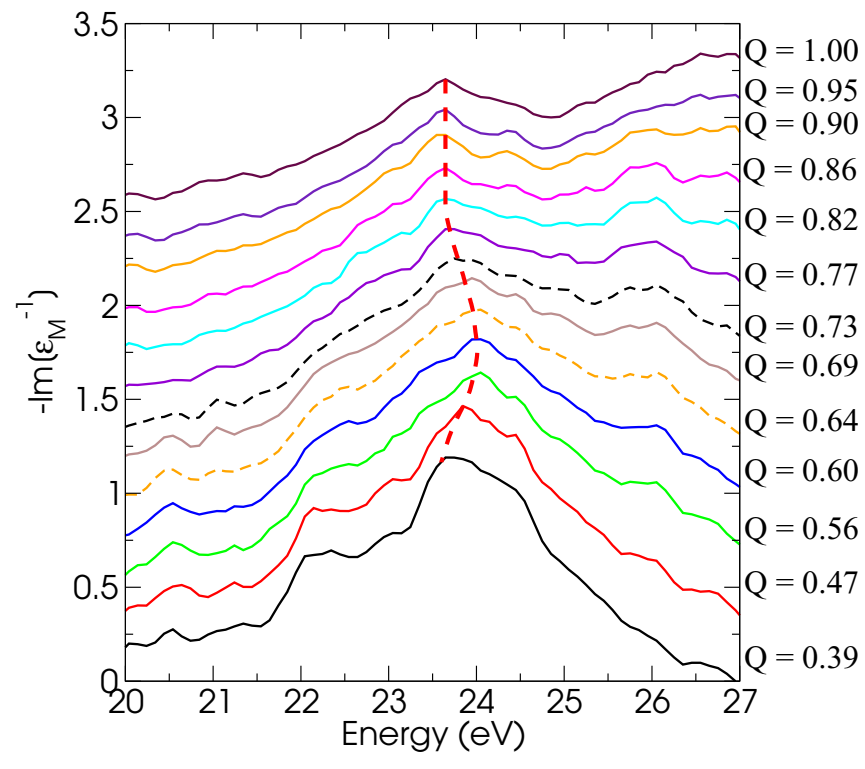

FIG. 4. (Color online) RPA loss function of $\mathrm{Cu}_{0.2} \mathrm{NbS}_{2}$ evaluated at $\mathbf{Q}$ (in $\AA^{-1}$ units) along the $c$ axis with a broadening of $0.1 \mathrm{eV}$. The dashed red line is a guide for the eye.

\section{ANALYSIS OF THE SPECTRA}

To gain further insight into the nature of the collective excitations we now discuss the electronic structure of $\mathrm{NbS}_{2}$ in more detail. Similar considerations apply to $\mathrm{NbSe}_{2}$ as well, the electronic structure of the two systems being equivalent (compare Figs. 5 and 6). From the analysis of the density of states projected on atomic orbitals (see Fig. 5) we find that there is a strong hybridization between the $\mathrm{Nb} s d$ and $\mathrm{S} p_{x y}$ orbitals which are responsible for the formation of covalent $\mathrm{Nb}-\mathrm{S}$ bonds. In total there are 17 valence electrons per formula unit. The covalent in-plane $\sigma$ bonds are made up of 10 electrons. Among the remaining 7 electrons, one occupies the $\mathrm{Nb} d_{z}$ orbital and determines the metallic character of the system, while the remaining 6 electrons are distributed among the $\mathrm{S}$ atoms and are responsible for the interlayer van der Waals forces. In particular 2 electrons occupy the $p_{z}$ state which is close in energy to the metallic $d_{z}$ state, while the remaining

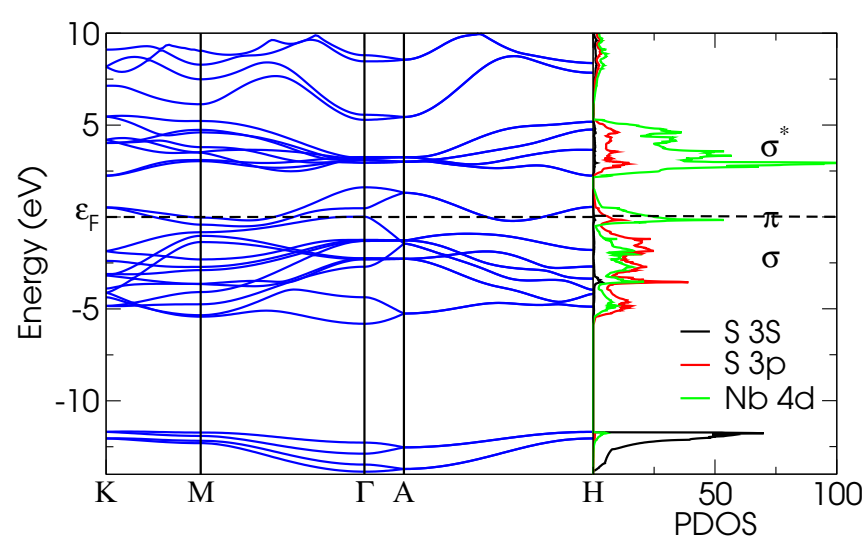

FIG. 5. (Color online) Band structure and projected density of states of $\mathrm{NbS}_{2}$. The zero of the vertical axis indicates the Fermi level. 


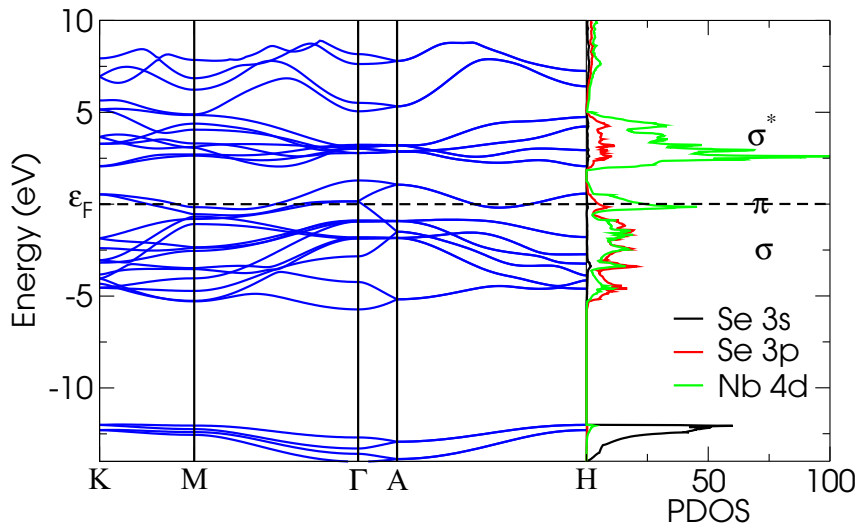

FIG. 6. (Color online) Same as Fig. 5 for $\mathrm{NbSe}_{2}$.

electrons give rise to two nonbonding $s$ states below the $\sigma$ bands. Thus, in analogy with graphite the electronic band structure of TMD can be characterized by $\pi$ and $\sigma$ states $[7,40]$. The latter are responsible for in-plane covalent bonds and cause a band gap opening in the band structure. The former involving the $p_{z}$ and $d_{z}$ states of $\mathrm{S}$ and $\mathrm{Nb}$ atoms respectively are responsible for the interlayer forces and are located inside the $\sigma-\sigma^{*}$ band gap. Therefore, from this analysis of the band structure we can identify three kinds of interband transitions: the low-energy $\pi-\pi^{*}$ transitions and the overlapping $\sigma-\sigma^{*}$ and $\pi-\sigma^{*}$ transitions at higher energy. With this picture in mind we now come back to our spectra. The layered structure of TMD gives rise to an anisotropic dielectric function. Figure 7(a) shows the real and imaginary parts of the dielectric function, $\epsilon_{M}=\epsilon_{1}+i \epsilon_{2}$, evaluated neglecting LFE for small $\mathbf{Q}$ parallel and perpendicular to the layers ("in-plane" and "out-of-plane," respectively). Besides the intraband peak (not shown), the in-plane $\epsilon_{2}$ [black line in Fig. 7(a)] is characterized by two main features at 2.9 and $4.6 \mathrm{eV}$ related to $\pi-\pi^{*}$ and $\sigma-\sigma^{*}$ transitions respectively. In the out-of-plane $\epsilon_{2}$ [red line in Fig. 7(a)] the first peak is suppressed and the oscillator strength is transferred

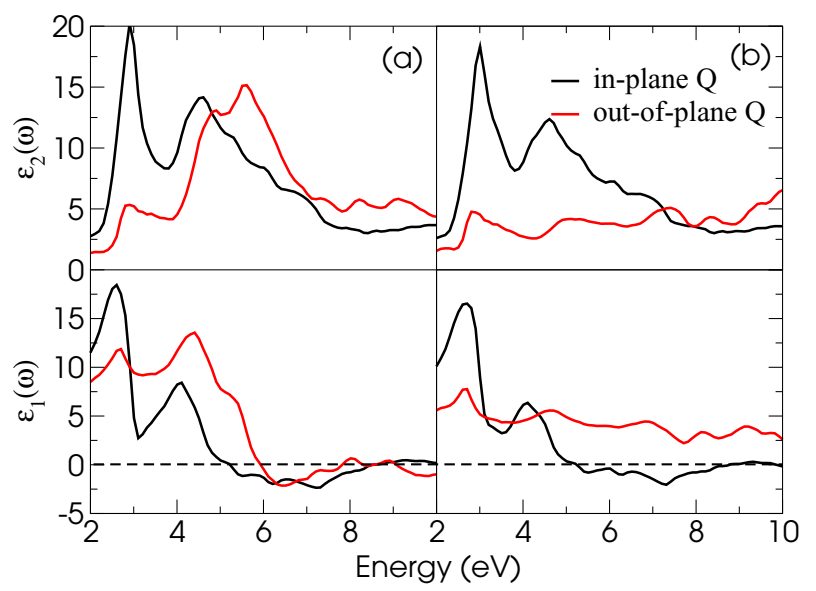

FIG. 7. (Color online) Real and imaginary part of the macroscopic dielectric function, $\epsilon_{M}=\epsilon_{1}+i \epsilon_{2}$, of $\mathrm{Cu}_{0.2} \mathrm{NbS}_{2}$ evaluated at $\mathbf{Q}=0.09 \AA^{-1}$ with a broadening of $0.1 \mathrm{eV}$ (a) neglecting and (b) taking into account LFE.

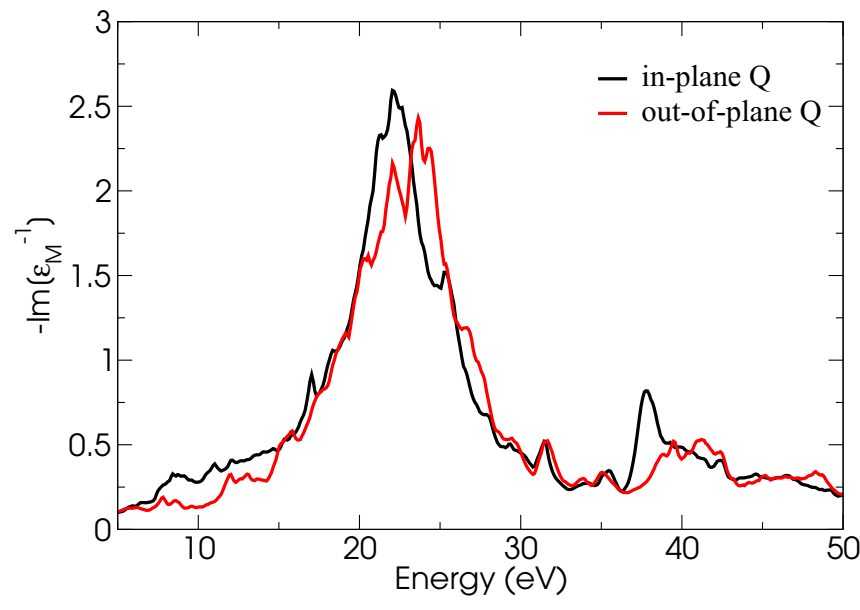

FIG. 8. (Color online) Comparison between the RPA spectra of $\mathrm{Cu}_{0.2} \mathrm{NbS}_{2}$ evaluated for in-plane and out-of-plane momentum transfer $\mathbf{Q}=0.09 \AA^{-1}$ with a broadening of $0.1 \mathrm{eV}$.

to higher-energy interband transitions between 4.7 and $5.5 \mathrm{eV}$, which involve mainly $\pi$ and $\sigma^{*}$ bands.

Thus, like in graphite [41], $\pi-\pi^{*}$ and $\sigma-\sigma^{*}$ transitions dominate the optical spectrum for in-plane $\mathbf{Q}$, while for out-ofplane $\mathbf{Q}$ the main contribution comes from $\pi-\sigma^{*}$ transitions. The main difference with graphite is that in this case, since $\pi$ and $\sigma$ bands overlap between them, $\sigma-\sigma^{*}$ and $\pi-\sigma^{*}$ transitions are not completely separated and are very close to the $\pi-\pi^{*}$ ones. This has important consequences on the shape of the loss function. In fact, contrarily to what happens in graphite, due to the proximity of the $\sigma$ states, $\pi$ electrons are not able to sustain collective excitations alone. On the other hand $\epsilon_{1}$ goes to zero at a frequency larger than the energy of the $\sigma-\sigma^{*}\left(\pi-\sigma^{*}\right)$ electron-hole pairs giving rise to a plasmon at $9 \mathrm{eV}$ [see lower panel in Fig. 7(a)]. As a consequence, this collective excitation is not a pure $\pi$ plasmon $[14,15]$ as in graphite, but it presents $\sigma$ character as well. The only collective excitation involving solely $\pi$ states in TMD is hence the intraband plasmon originating from the $\mathrm{Nb} d_{z}$ orbital [20]. The anisotropy in the dielectric function is also clearly visible when LFE are taken into account [41] since the induced charge is specially more homogeneous in the plane of the layers than in the direction perpendicular to them. In fact, we find that LFE are almost negligible for in-plane $\epsilon_{2}$ [compare black lines in Figs. 7(a) and 7(b)] while they are stronger for out-of-plane $\epsilon_{2}$ [compare red lines in Figs. 7(a) and 7(b)], where the $\pi-\sigma^{*}$ peak is strongly suppressed and blueshifted towards the plasmon energy. These depolarization effects on $\epsilon_{2}$ affect also $\epsilon_{1}$ through the Kramers-Kronig relations [see the lower panel in Fig. 7(b)]. Due to the suppression of $\epsilon_{2}$, the out-of-plane $\epsilon_{1}$ remains positive in the low-energy region of the spectrum and thus the plasmon at $9 \mathrm{eV}$ is completely suppressed. In other words, the damping induced by $\pi-\sigma^{*}$ electron-hole pairs is so strong that the system is not able to sustain collective excitations at low energy for out-of-plane $\mathbf{Q}$ : the $9 \mathrm{eV}$ plasmon is much more visible for in-plane $\mathbf{Q}$ (see Fig. 8). The anisotropy affects also the higher-energy part of the spectrum. In fact, the depolarization effect induced by LFE on the semicore peak is weaker for in-plane $\mathbf{Q}$. As a consequence, for in-plane $\mathbf{Q}$ 


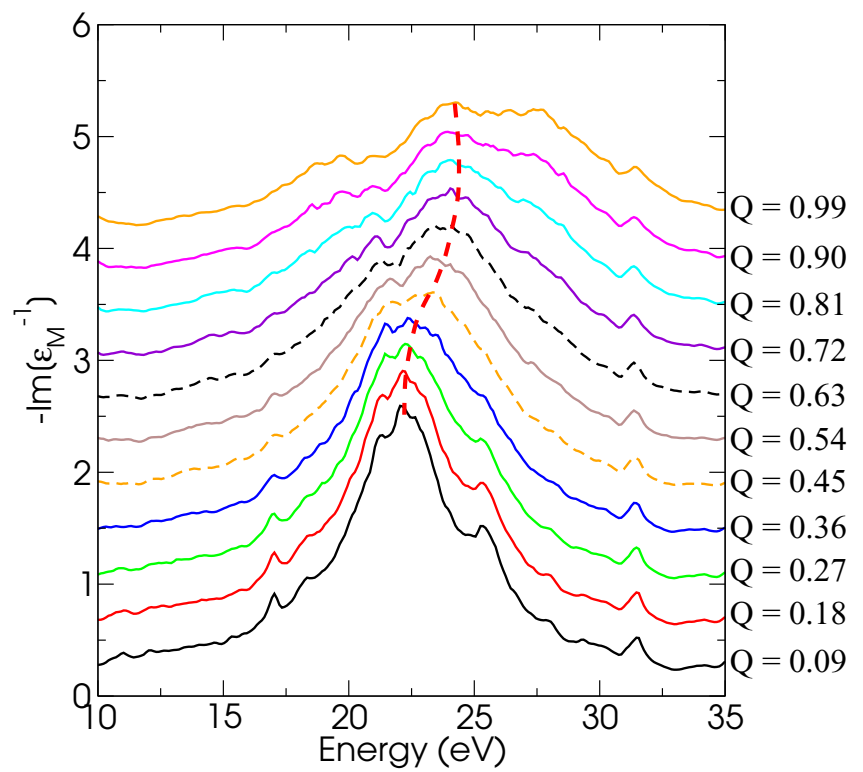

FIG. 9. (Color online) RPA spectra of $\mathrm{Cu}_{0.2} \mathrm{NbS}_{2}$ evaluated for in-plane $\mathbf{Q}$ (in $\AA^{-1}$ units) with a broadening of $0.1 \mathrm{eV}$. The dashed red line is a guide for the eye.

the interband transitions involving semicore states are more efficient in screening the main plasmon that is redshifted by about $2 \mathrm{eV}$ with respect to what is observed for out-of-plane $\mathbf{Q}$ (compare black and red lines in Fig. 8). Finally, we discuss the effect of the anisotropy on the dispersion of the high-energy plasmon. As we have seen in the previous section, the lack of dispersion for out-of-plane $\mathbf{Q}$ is related to the activation of high-energy interband transitions at large momentum transfer, which screen the plasmon frequency. From our analysis of the electronic structure, we can ascribe these transitions to electron-hole pairs involving the low energy nonbonding $\mathrm{S} s$ orbitals and high-energy $\sigma^{*}$ states. They are dipole forbidden for out-of-plane $\mathbf{Q}$, but are activated by multipole terms as $\mathbf{Q}$ increases. The effect related to the activation of these multipole excitations is instead not visible for the in-plane polarizations, since in this case the various transitions are already allowed in the dipole limit. As a consequence they do not affect the inplane plasmon dispersion which remains positive (see Fig. 9).

\section{CONCLUSIONS}

In conclusion, combining state-of-the-art IXS experiments and TDDFT calculations we have investigated the collective excitations in two prototypical TMD, namely $\mathrm{Cu}_{0.2} \mathrm{NbS}_{2}$ and $\mathrm{NbSe}_{2}$. Provided that the $\mathrm{Nb}$ semicore states are explicitly taken into account in the calculation, we find good agreement between experiment and theory already at the level of the random-phase approximation. In particular, besides the intraband plasmon [20] at $\sim 1 \mathrm{eV}$, also at high energies these materials show very similar loss spectra, characterized by two plasmons at $\sim 9 \mathrm{eV}$ and at $\sim 23 \mathrm{eV}$. From the detailed analysis of the band structure we have identified a $\pi+\sigma$ character for both plasmons. We have discussed in detail the role the anisotropy associated to the layered structure of TMD, also in connection with the different effect of crystal local fields for a momentum transfer $\mathbf{Q}$ within the layers or normal to them. The $9 \mathrm{eV}$ plasmon is visible for in-plane $\mathbf{Q}$, while for $\mathbf{Q}$ parallel to the $c$ axis it is strongly damped by single-particle excitations, as a result of which it is completely washed out in the electron hole continuum. The $23 \mathrm{eV}$ plasmon has a positive dispersion for in-plane $\mathbf{Q}$, while for for $\mathbf{Q}$ parallel to the $c$ axis it has an oscillating behavior. This is due to the activation at large $\mathbf{Q}$ of dipole-forbidden interband transitions at energy above the plasmon frequency.

\section{ACKNOWLEDGMENTS}

We would like to thank to Pasi Heikkilä and the Department of Geosciences and Geography, University of Helsinki, for the use of the electron probe microanalyzer. We are grateful to Mikko Hakala, Marco Grioni and Eugenio Coronado for invaluable discussions. We acknowledge financial support from the European Research Council Advanced Grant DYNamo (ERC-2010-AdG-267374), Spanish grant (2010-21282-C0201), Grupos Consolidados UPV/EHU del Gobierno Vasco (IT578-13), European Commission project CRONOS (Grant No. 280879-2). This research was also supported by a Marie Curie FP7 Integration Grant within the 7th European Union Framework Programme, and by Generalidad Valenciana (ISIC Nano program). Computational time was granted by GENCI (Project No. 544) and BSC "Red Espanola de Supercomputacion." S.H., K.O.R. and C.J.S. were supported by the Academy of Finland (projects 1256211, 1254065, 1259526) and University of Helsinki Research Funds (project 490076).
[1] H. Park, J. Park, A. K. L. Lim, E. H. Anderson, A. P. Alivisatos, and P. L. McEuen, Nature (London) 407, 57 (2000).

[2] A. I. Yanson, G. R. Bollinger, H. E. van den Brom, N. Agrait, and J. M. van Ruitenbeek, Nature (London) 395, 783 (1998).

[3] C. Lee, Q. Y. Li, W. Kalb, X. Z. Liu, H. Berger, R. W. Carpick, and J. Hone, Science 328, 76 (2010).

[4] K. S. Novoselov, A. K. Geim, S. V. Morozov, D. Jiang, Y. Zhang, S. V. Dubonos, I. V. Grigorieva, and A. A. Firsov, Science 306, 666 (2004).

[5] M. I. Katsnelson, Mater. Today 10, 20 (2007).
[6] A. H. Castro Neto, F. Guinea, N. M. R. Peres, K. S. Novoselov, and A. K. Geim, Rev. Mod. Phys. 81, 109 (2009).

[7] J. Wilson and A. Yoffe, Adv. Phys. 18, 193 (1969).

[8] J. N. Coleman et al., Science 331, 568 (2011).

[9] C. Ataca, H. Sahin, and S. Ciraci, J. Phys. Chem. C 116, 8983 (2012).

[10] K. Rossnagel, J. Phys.: Condens. Matter 23, 213001 (2011).

[11] A. Splendiani, L. Sun, Y. Zhang, T. Li, J. Kim, C. Y. Chim, G. Galli, and F. Wang, Nano Lett. 10, 1271 (2010).

[12] K. F. Mak, C. Lee, J. Hone, J. Shan, and T. F. Heinz, Phys. Rev. Lett. 105, 136805 (2010). 
[13] B. Radisavljevic, A. Radenovic, J. Brivio, V. Giacometti, and A. Kis, Nat. Nanotechnol. 6, 147 (2011).

[14] W. Y. Liang and S. L. Cundy, Philos. Mag. 19, 1031 (1969).

[15] K. Zeppenfeld, Opt. Commun. 1, 377 (1970).

[16] G. Bell and W. Y. Liang, Adv. Phys. 25, 53 (1976).

[17] J. van Wezel, R. Schuster, A. König, M. Knupfer, J. van den Brink, H. Berger, and B. Büchner, Phys. Rev. Lett. 107, 176404 (2011).

[18] R. Schuster, R. Kraus, M. Knupfer, H. Berger, and B. Büchner, Phys. Rev. B 79, 045134 (2009).

[19] M. N. Faraggi, A. Arnau, and V. M. Silkin, Phys. Rev. B 86, 035115 (2012).

[20] P. Cudazzo, M. Gatti, and A. Rubio, Phys. Rev. B 86, 075121 (2012).

[21] R. Hambach, C. Giorgetti, N. Hiraoka, Y. Q. Cai, F. Sottile, A. G. Marinopoulos, F. Bechstedt, and L. Reining, Phys. Rev. Lett. 101, 266406 (2008).

[22] E. Runge and E. K. U. Gross, Phys. Rev. Lett. 52, 997 (1984).

[23] A. M. Gabovich, A. I. Voitenko, and M. Ausloos, Phys. Rep. 367, 583 (2002).

[24] D. S. Inosov, V. B. Zabolotnyy, D. V. Evtushinsky, A. A. Kordyuk, B. Büchner, R. Follath, H. Berger, and S. V. Borisenko, New J. Phys. 10, 125027 (2008).

[25] D. V. Evtushinsky, A. A. Kordyuk, V. B. Zabolotnyy, D. S. Inosov, B. Büchner, H. Berger, L. Patthey, R. Follath, and S. V. Borisenko, Phys. Rev. Lett. 100, 236402 (2008).

[26] F. Lévy and H. Berger, J. Cryst. Growth 61, 61 (1983).

[27] G. Onida, L. Reining, and A. Rubio, Rev. Mod. Phys. 74, 601 (2002).

[28] X. Gonze, G.-M. Rignanese, M. Verstraete, J.-M. Beuken, Y. Pouillon, R. Caracas, F. Jollet, M. Torrent, G. Zerah, M. Mikami, Ph. Ghosez, M. Veithen, J.-Y. Raty, V. Olevano, F. Bruneval, L. Reining, R. Godby, G. Onida, D. R. Hamann, and D. C. Allan, Zeit. Kristallogr. 220, 558 (2005).

[29] E. Moncton, J. D. Axe, and F. J. Di Salvo, Phys. Rev. B 16, 801 (1977); W. G. Fisher and M. J. Sienko, Inorg. Chem. 19, 39 (1980); A. Meetsma, G. A. Wiegers, R. J. Haange, and J. L. de Boer, Acta Crystallogr. Sect. C 46, 1598 (1990).
[30] N. Troullier and J. L. Martins, Phys. Rev. B 43, 1993 (1991); C. Hartwigsen, S. Goedecker, and J. Hutter, ibid. 58, 3641 (1998).

[31] A. Marini, C. Hogan, M. Gruning, and D. Varsano, Comput. Phys. Commun. 180, 1392 (2009).

[32] The $c$ lattice parameters were determined in situ to be 12.11 and $12.54 \AA$ for the samples of $\mathrm{Cu}_{0.2} \mathrm{NbS}_{2}$ and $\mathrm{NbSe}_{2}$ respectively. For the calculation we used $c=11.95 \AA$ for $\mathrm{Cu}_{0.2} \mathrm{NbS}_{2}$ and $c=12.55 \AA$ for $\mathrm{NbSe}_{2}$, as in Ref. [29].

[33] H.-Ch. Weissker, J. Serrano, S. Huotari, F. Bruneval, F. Sottile, G. Monaco, M. Krisch, V. Olevano, and L. Reining, Phys. Rev. Lett. 97, 237602 (2006).

[34] M. Cazzaniga, H.-Ch. Weissker, S. Huotari, T. Pylkkänen, P. Salvestrini, G. Monaco, G. Onida, and L. Reining, Phys. Rev. B 84, 075109 (2011).

[35] S. Huotari, M. Cazzaniga, H.-Ch. Weissker, T. Pylkkänen, H. Müller, L. Reining, G. Onida, and G. Monaco, Phys. Rev. B 84, 075108 (2011).

[36] N. Vast, L. Reining, V. Olevano, P. Schattschneider, and B. Jouffrey, Phys. Rev. Lett. 88, 037601 (2002).

[37] P. Johari and V. B. Shenoy, ACS Nano 5, 5903 (2011); P. Cudazzo, M. Gatti, and A. Rubio, New J. Phys. 15, 125005 (2013).

[38] M. Gatti, Ph.D. thesis, Ecole Polytechnique, 2007, http://etsf.polytechnique.fr/sites/default/files/users/ matteo/matteo_thesis.pdf; L. Dash, F. Bruneval, V. Trinité, N. Vast, and L. Reining, Comput. Mater. Sci. 38, 482 (2007); S. Huotari, J. A. Soininen, G. Vankó, G. Monaco, and V. Olevano, Phys. Rev. B 82, 064514 (2010).

[39] F. Aryasetiawan and K. Karlsson, Phys. Rev. Lett. 73, 1679 (1994); P. Cudazzo, M. Gatti, F. Roth, B. Mahns, M. Knupfer, and A. Rubio, Phys. Rev. B 84, 155118 (2011).

[40] L. F. Mattheis, Phys. Rev. B 8, 3719 (1973).

[41] A. G. Marinopoulos, L. Reining, V. Olevano, A. Rubio, T. Pichler, X. Liu, M. Knupfer, and J. Fink, Phys. Rev. Lett. 89, 076402 (2002); A. G. Marinopoulos, L. Reining, A. Rubio, and V. Olevano, Phys. Rev. B 69, 245419 (2004). 